\title{
Observation and Inpatient Status: Clinical Impact of the 2-Midnight Rule
}

\author{
Ann M. Sheehy, MD, MS ${ }^{1 \star}$, Bartho Caponi, MD², Sreedevi Gangireddy, MD², Azita G. Hamedani, MD, MPH³, \\ Jeffrey J. Pothof, MD³ , Eric Siegal, MD², Ben K. Graf, MD
}

\begin{abstract}
${ }^{1}$ Department of Medicine, Division of Hospital Medicine, University of Wisconsin School of Medicine and Public Health, Madison, Wisconsin; ${ }^{2}$ Department of Medicine, Division of Hospital Medicine, University of Wisconsin, School of Medicine and Public Health, Madison, Wisconsin; ${ }^{3}$ Department of Medicine, Division of Emergency Medicine, University of Wisconsin, School of Medicine and Public Health, Madison, Wisconsin; ${ }^{4}$ Aurora Health Care Critical Care Service, Milwaukee, WI, and Department of Medicine, University of Wisconsin School of Medicine and Public Health, Madison, Wisconsin; ${ }^{5}$ Department of Orthopedics and Rehabilitation, University of Wisconsin, School of Medicine and Public Health, Madison, Wisconsin.
\end{abstract}

BACKGROUND: In response to growing concern over frequency and duration of observation encounters, the Centers for Medicare and Medicaid Services enacted a rules change on October 1, 2013, classifying most hospital encounters of $<2$ midnights as observation, and those $\geq 2$ midnights as inpatient. However, limited data exist to predict the impact of the new rule.

OBJECTIVE: To answer the following: (1) Will the rule reduce observation encounter frequency? (2) Are short-stay ( $<2$ midnights) inpatient encounters often misclassified observation encounters? (3) Do 2 midnights separate distinct clinical populations, making this rule logical? (4) Do nonclinical factors such as time of day of admission impact classification under the rule?

DESIGN, SETTING AND PATIENTS: Retrospective descriptive study of all observation and inpatient encounters initiated between January 1, 2012 and February 28, 2013 at a Midwestern academic medical center.
MEASUREMENTS: Demographics, insurance type, and characteristics of hospitalization were abstracted for each encounter.

RESULTS: Of 36,193 encounters, 4,769 (13.2\%) were observation. Applying the new rules predicted a net loss of $14.9 \%$ inpatient stays; for Medicare only, a loss of $7.4 \%$. Less than 2-midnight inpatient and observation stays were different, sharing only 1 of 5 top International Classification of Diseases, 9th Revision (ICD-9) codes, but for encounters classified as observation, 4 of 5 top ICD-9 codes were the same across the length of stay. Observation encounters starting before 8:00 AM less commonly spanned 2 midnights (13.6\%) than later encounters (31.2\%).

CONCLUSIONS: The 2-midnight rule adds new challenges to observation and inpatient policy. These findings suggest a need for rules modification. Journal of Hospital Medicine 2014;9:203-209. (c) 2014 Society of Hospital Medicine
Observation hospitalization is a growing phenomenon in the United States healthcare system. ${ }^{1}$ For Medicare beneficiaries, observation encounters increased 33.6\% from 2004 to 2011, with inpatient encounters decreasing by $7.8 \%$ over the same period. ${ }^{2}$

Observation length of stay has also increased. Medicare states that observation care should typically last $<24$ hours, and in only "rare and exceptional" cases exceed 48 hours. We and others have showed that observation stays, on average, do not meet this definition. ${ }^{1,3}$ At our institution, historically less than half of

\footnotetext{
*Address for correspondence and reprint requests: Ann M. Sheehy, MD, Department of Medicine, Division of Hospital Medicine, University of Wisconsin School of Medicine and Public Health, 1685 Highland Avenue, MFCB 3126, Madison, WI 53705; Telephone: 608-262-2434; Fax: 608265-1420; E-mail: asr@medicine.wisc.edu

Additional Supporting Information may be found in the online version of this article.

This article was published online on 14 February 2014. The editor added a comment subsequent to Early View publication. This notice is included in the online and print versions to indicate that both have been corrected 14 March 2014.

Received: October 21, 2013; Revised: December 23, 2013; Accepted: January 9, 2014

2014 Society of Hospital Medicine DOI 10.1002/jhm.2163

Published online in Wiley Online Library (Wileyonlinelibrary.com).
}

observation encounters discharge in under 24 hours, and 1 in 6 stay longer than 48 hours. $^{3}$

The Centers for Medicare and Medicaid Services (CMS) issued a rules change effective October 1, 2013, in response to "concern about recent increases in the length of time that CMS beneficiaries spend as hospital outpatients receiving observation services...."4 These rules shifted observation determination from clinical criteria, such as InterQual, ${ }^{5}$ to a time-based rule that hinges on a 2-midnight cut point. Patients staying $<2$ midnights, with few exceptions, are now observation, and those staying $\geq 2$ midnights are inpatients. This is important, as patients hospitalized as observation are technically outpatients, not covered by Medicare Part A hospital insurance, and ineligible for skilled nursing facility benefits. ${ }^{3,6}$

Although challenges with observation status in general are well described, ${ }^{1,3,7}$ the potential impact of the 2 -midnight rule is not fully known. The purpose of our descriptive study was to examine how the new rules, retrospectively applied to recent encounters, would affect our hospital encounters, with a separate analysis of Medicare encounters and adult general medicine encounters. First, as CMS predicted "a net shift from outpatient to inpatient stays" 8 under the new 
rule, we attempted to determine whether this rule would actually reduce observation encounter frequency. Second, as CMS has cited "persistently large improper payment rates in short-stay hospital inpatient claims" 4 and intends to audit $<2$-midnight inpatient encounters under the assumption that many are misclassified observation stays, ${ }^{4,8,9}$ we sought to determine if short-stay inpatient and observation encounters were truly the same. Third, as insurance coverage will change based on the 2-midnight cut point, we sought to determine whether 2 midnights separated distinct clinical populations within observation status, making the rule logical and fair. Finally, we sought to determine whether external factors, such as time of admission, day of week of admission, and transfer to our institution would impact how patients may be classified under the new rule.

\section{METHODS}

\section{Study Population}

Our methods have been described previously ${ }^{3}$ with the exception of the updated dates of inclusion. Briefly, we analyzed all observation and inpatient encounters at University of Wisconsin Hospital and Clinics (UWHC) and the adjacent American Family Children's Hospital, a tertiary care referral academic medical center in Madison, Wisconsin, ${ }^{10}$ with start date between January 1, 2012 and February 28, 2013. Six encounters with length of stay (LOS) $>6$ months were censored as they were not discharged by the time of data abstraction, and 3 encounters were removed due to erroneous encounter discharge dates. Patients with all insurance types were included in anticipation that commercial payors will follow Medicare rules changes. The University of Wisconsin (UW) Health Sciences Institutional Review Board approved the study.

\section{Data Sources}

Data were abstracted from the UW Health Sciences electronic medical record and ancillary data systems by the UWHC Business Planning and Analysis Department. Variables included demographics (age, sex, ethnicity), insurance type, and characteristics of hospitalization (admission service, day of week encounter began, acute/ unscheduled presentation, International Classification of Diseases, 9th Revision [ICD-9] codes, LOS, transfer from other hospital). We considered inpatient admission start time to be departure from the emergency department (ED) or arrival at our hospital if the encounter was a transfer from another facility. Observation start time also hinged on arrival at our hospital if the encounter was transferred from another facility, but for observation encounters arriving from the ED, the ED rooming time was used as the encounter start based on conservative interpretation of CMS rules for observation start time under the 2-midnight rule. ${ }^{11}$ Discharge time for all encounters was considered physical discharge from the ward. Observation status decisions were made by the admitting physician, with as-needed guidance by case management staff and utilization review physicians using InterQual Criteria (McKesson Corp, San Francisco, CA), ${ }^{5}$ a reference historically used by CMS auditors to determine status.

\section{Evaluation of the 2-Midnight Rule}

All encounters were stratified based on a stay of $<2$ midnights or $\geq 2$ midnights. Because general medicine patients comprise the bulk of observation patients, ${ }^{3}$ the subset of encounters occurring on the hospital's 4 hospitalist medicine services, 3 general medicine resident services, and 1 adult nonobstetrics family medicine service, collectively termed adult general medicine, were also evaluated separately. Medicare encounters were also evaluated separately.

We first specifically compared $<2$-midnight inpatient encounters and $\geq 2$-midnight observation encounters during the study period to determine the net number of encounters that would lose inpatient status $(<2$-midnight inpatient encounters) and that would gain inpatient status $(\geq 2$-midnight observation encounters) under the new rules. Subtracting the absolute number of $<2$-midnight inpatient encounters from the $\geq 2$-midnight observation encounters results in the net loss or gain of inpatient encounters, assuming LOS does not change. Second, we compared ICD9 codes between $<2$-midnight inpatient encounters and observation encounters to determine if these 2 groups were clinically distinguishable. Third, we compared diagnosis codes between observation encounters lasting $<2$ midnights and $\geq 2$ midnights to establish whether the 2-midnight cut point defines distinct patient groups within observation. Finally, we evaluated all observation encounters to determine whether the time of admission, the day of admission (weekday vs weekend), or whether the encounter had been transferred from another facility impacted encounter categorization under the new rules.

\section{Statistical Methods}

Descriptive statistics were used in this study, with data largely summarized as number and percent. When appropriate, mean and standard deviation were used to describe central tendency and dispersion.

\section{RESULTS \\ Characteristics of Inpatient and Observation Encounters}

Of the 36,193 total hospital encounters during the study period, 4769 (13.2\%) were classified as observation encounters. Of 8510 adult general medicine encounters, $2443(28.7 \%)$ were observation. Adult general medicine observation encounters accounted for $51.2 \%$ of all observation encounters for the hospital. A total of $9.0 \%$ of our observation encounters were transferred from another institution (Table 1). 
TABLE 1. Characteristics of Inpatient and Observation Encounters $(\mathrm{N}=36,193)$

\begin{tabular}{lcc}
\hline & $\begin{array}{c}\text { Inpatient, } \mathrm{n}=31,424 \\
(86.8 \%)\end{array}$ & $\begin{array}{c}\text { Observation, } \mathrm{n}=4,769 \\
(13.2 \%)\end{array}$ \\
\hline Demographics & & \\
Female & $15,083(48.0 \%)$ & $2,321(48.7 \%)$ \\
Age, y, mean (SD) & $49.2(23.6)$ & $49.4(25.4)$ \\
Has primary care provider & $27,378(87.1 \%)$ & $4,152(87.1 \%)$ \\
Ethnicity & & \\
Caucasian & $27,145(86.4 \%)$ & $3,880(81.4 \%)$ \\
Non-Caucasian & $3,478(11.1 \%)$ & $739(15.5 \%)$ \\
Unknown & $801(2.5 \%)$ & $150(3.1 \%)$ \\
Characteristics of hospitalization & & \\
Day of admission & & \\
Weekend (Saturday-Sunday) & $5,058(16.1 \%)$ & $1,129(23.7 \%)$ \\
Weekday (Monday-Friday) & $26,360(83.9 \%)$ & $3,640(76.3 \%)$ \\
Transfer from other institution & $6,191(19.7 \%)$ & $427(9.0 \%)$ \\
Acute/unscheduled & $21,150(67.3 \%)$ & $4,479(93.9 \%)$ \\
Service of admission & & \\
Adult general medicine & $6,067(19.3 \%)$ & $2,443(51.2 \%)$ \\
Adult surgery & $13,625(43.4 \%)$ & $856(17.9 \%)$ \\
Adult subspecialty nonsurgery & $7,432(23.7 \%)$ & $802(16.8 \%)$ \\
Pediatrics & $4,300(13.7 \%)$ & $668(14.0 \%)$ \\
Insurance & & \\
Medicare & $11,719(37.3 \%)$ & $1,846(38.7 \%)$ \\
Medicaid & $3,642(11.6 \%)$ & $658(13.8 \%)$ \\
Commercial & $13,321(42.4 \%)$ & $1,817(38.1 \%)$ \\
Other & $1,665(5.3 \%)$ & $184(3.9 \%)$ \\
None & $1,077(3.4 \%)$ & $264(5.5 \%)$ \\
\hline NOTE: All data are number (\%) unless otherwise specified. Abbreviations: SD, standard deviation. \\
\end{tabular}

A total of $1442(4.0 \%)$ encounters changed status during the study period, with $606(42.0 \%)$ having changed from inpatient to observation and considered observation, and $836(58.0 \%)$ having changed from observation to inpatient and considered inpatient.

\section{Impact of the 2-Midnight Rule on Number of Observation and Inpatient Encounters}

Among all encounters, the 2-midnight rule would result in a net transition of $14.9 \%$ of inpatient encounters to observation. Considering only Medicare encounters, the net transition would be $7.4 \%$ inpatient encounters to observation. Within adult general medicine patients, the parallel changes would be $-2.2 \%$ (all insurance types) and a gain of $2.4 \%$ (Medicare only) (Table 2).

Encounters including surgical procedures on the socalled inpatient-only list will remain inpatient regardless of LOS. As we could not identify such encounters, we tested removal of all surgical stays under the overly conservative assumption that all short stay surgical patients would remain inpatient. Of 21,712 nonsurgical encounters that remained, there were $4074<2$-midnight inpatient encounters and 1146 $\geq 2$-midnight observation encounters, yielding a net transition of $2928(13.5 \%)$ inpatient encounters to observation encounters. Medicare encounters accounted for 8240 of these $21,712(38.0 \%)$ nonsurgical encounters, with $1105<2$-midnight inpatient
TABLE 2. Impact of the 2-Midnight Rule on Number of Observation and Inpatient Encounters

$(\mathrm{N}=36,193)$

\begin{tabular}{lcc}
\hline & $\begin{array}{c}\text { Inpatient, } n=31,424 \\
(86.8 \%)\end{array}$ & $\begin{array}{c}\text { Observation, } n=4,769 \\
(13.2 \%)\end{array}$ \\
\hline All encounters, $n=36,193$ & & \\
$<2$ Midnights & $6,723^{*}(21.4 \%)$ & $3,454(72.4 \%)$ \\
$\geq 2$ Midnights & $24,701(78.6 \%)$ & $1,315^{*}(27.6 \%)$ \\
Net change inpatient encounters & $-5,408 \dagger(-14.9 \%)$ & \\
Medicare encounters, $n=13,565$ & & \\
$<2$ Midnights & $1,728^{*}(14.7 \%)$ & $1,127(61.1 \%)$ \\
$\geq 2$ Midnights & $9,991(85.3 \%)$ & $719^{*}(38.9 \%)$ \\
Net change inpatient encounters & $-1,009 \dagger(-7.4 \%)$ & \\
All general medicine, $n=8,510$ & & \\
$<2$ Midnights & $1,114^{*}(18.4 \%)$ & $1,512(61.9 \%)$ \\
$\geq 2$ Midnights & $4,953(81.6 \%)$ & $931^{*}(38.1 \%)$ \\
Net change inpatient encounters & $-183 \dagger(-2.2 \%)$ & \\
Medicare general medicine, $n=4,571$ & & \\
$<2$ Midnights & $472^{*}(14.3 \%)$ & $690(54.2 \%)$ \\
$\geq 2$ Midnights & $2,827(85.7 \%)$ & $582^{*}(45.8 \%)$ \\
Net change inpatient encounters & $110 \dagger(2.4 \%)$ & \\
& &
\end{tabular}

NOTE: All data are number (\%). ${ }^{*}$ Numbers represent encounters affected by rules change (inpatients $<2$ midnights, observation $\geq 2$ midnights). †Net change inpatient encounters is calculated by subtracting the number of $<2$-midnight inpatient encounters (those encounters that would be observation under the new rules) from the number of $\geq 2$-midnight observation encounters (those encounters that would be inpatient under the new rules) for the total net change in inpatient encounters.

encounters and $653 \geq 2$-midnight observation encounters, yielding a net reclassification of 452 (5.5\%) Medicare nonsurgical inpatient encounters to observation encounters.

\section{Length of Stay and Diagnoses After Application of the 2-Midnight Rule to Inpatient and Observation Encounters}

Only 1 of the top 5 ICD-9 codes (code 427: cardiac dysrhythmias) was shared between the $\leq 2$-midnight inpatient encounters and any observation encounter group. When the same criteria were applied to adult general medicine encounters, none of the top 5 ICD-9 codes were shared (Table 3).

Including all observation encounters across LOS, 4 of the 5 top ICD-9 codes were the same for stays $<2$ midnights and $\geq 2$ midnights. The same was true for short- and long-stay general medicine observation encounters (Table 3).

For all observation encounters, 26.5\% (348/1315) of $\geq 2$-midnight encounters started on a weekend compared to $22.6 \%(781 / 3454)$ of $<2$-midnight encounters. For adult medicine observation, $28.0 \%$ (261/ 931) of $\geq 2$-midnight encounters started on a weekend compared to $21.0 \%(318 / 1512)$ of $<2$-midnight encounters (Table 3).

\section{Percentage of Observation Encounters Reaching $\geq 2$ Midnights Based on Time of Hospitalization}

Observation encounters starting before 8:00 AM spanned 2 midnights $13.6 \%$ of the time, and those encounters starting after 4:00 PM crossed 2 midnights $31.2 \%$ of the time. Two of the 3 top ICD-9 codes 


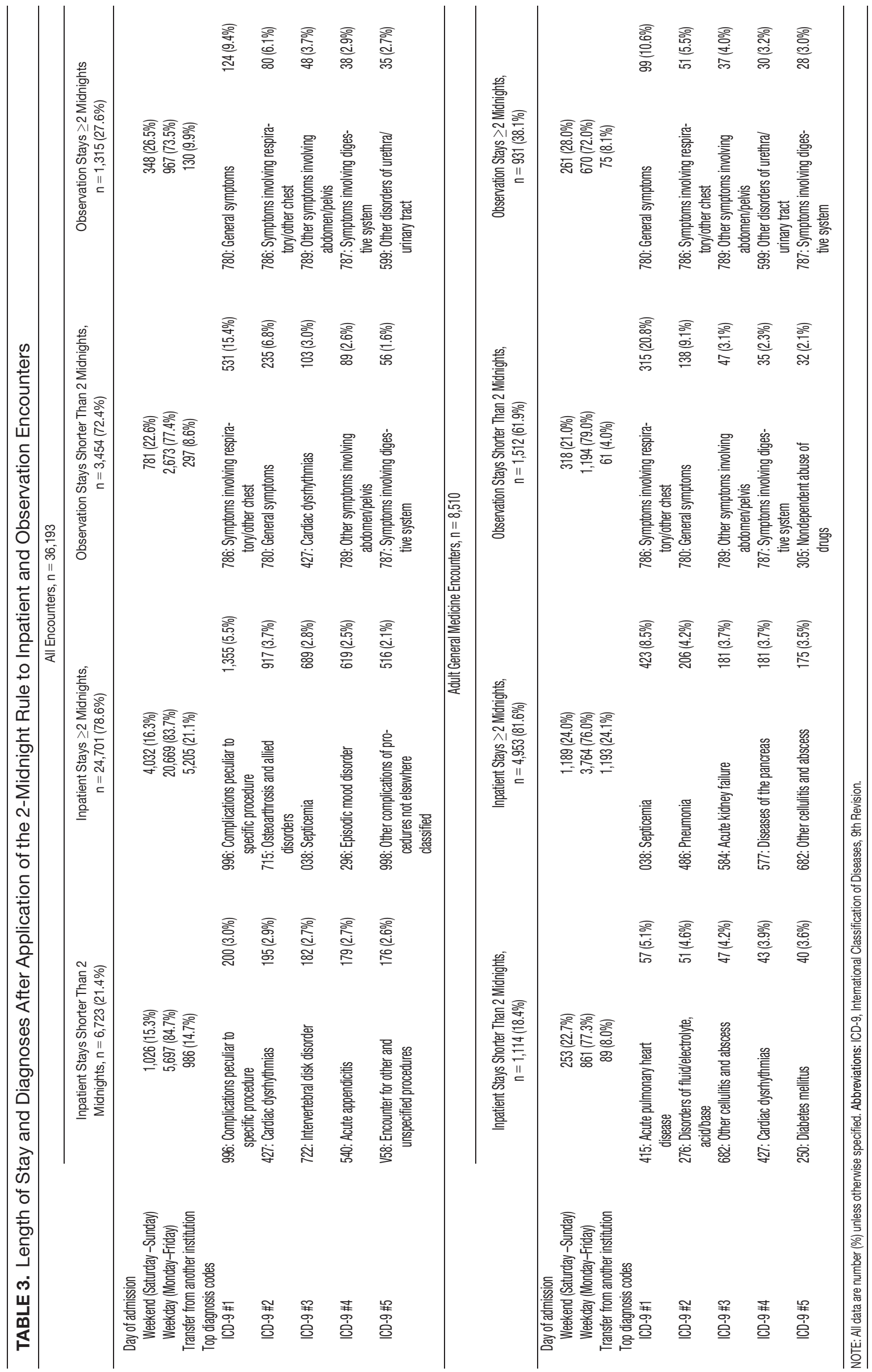




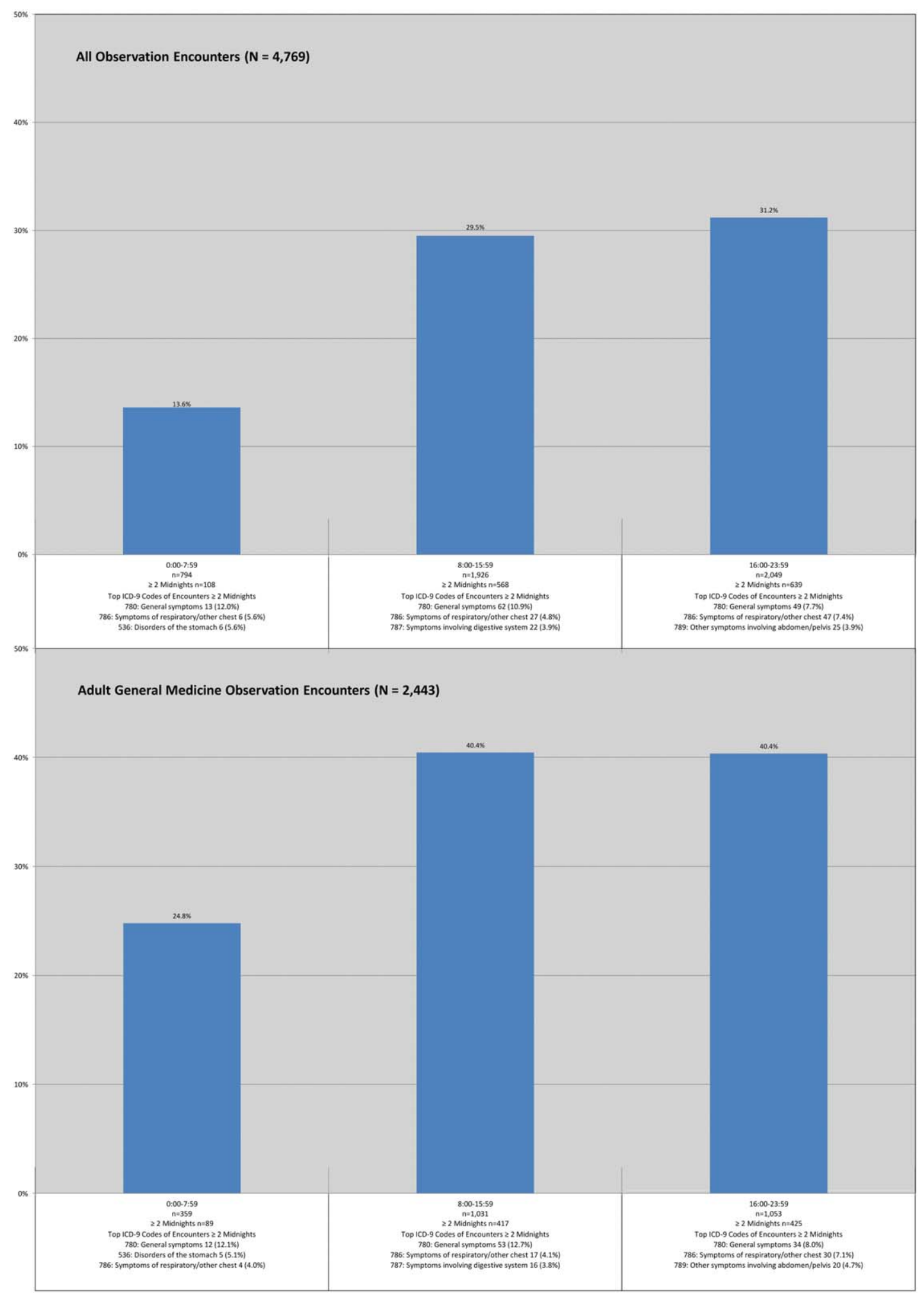

FIG. 1. Percentage of observation encounters reaching $\geq 2$ midnights based on time of hospitalization. Abbreviations: ICD-9, International Classification of Diseases, 9th Revision.

were the same across LOS, with similar findings for the adult general medicine-only group (Figure 1).

\section{DISCUSSION}

Although CMS predicts that more patients will be classified as inpatients under the new rule, we deter- mined the opposite, consistent with a recent report generated by the Office of the Inspector General (OIG) for 2012 Medicare beneficiaries. ${ }^{8}$ Our results did not change when we excluded all surgical encounters to account for possible exclusions based on the surgical inpatient-only list. Although a small percentage 
of Medicare adult general medicine patients may be reclassified as inpatients under the new rules, the net effect would be that many more hospital encounters will be billed under observation rather than inpatient status. These findings assume overall length of stay will remain unchanged under these rules, an assumption that may not hold true given the financial losses we predicted hospitals may face under this rule, ${ }^{12}$ and potential pressures on individual physicians providing patient care.

Medicare has prioritized auditing $<2$-midnight inpatient encounters under the assumption that many short inpatient encounters are actually misclassified observation encounters, ${ }^{4,8,9}$ prompting us to investigate whether this was the case in our patient population. Although it did not use ICD-9 diagnosis codes, the OIG report suggested that short-stay inpatients and observation patients may be clinically similar. ${ }^{8,13}$ Using ICD-9 codes, we found no overlap between the top ICD-9 codes for adult general medicine $<2$-midnight inpatient and observation encounters, and only 1 of 5 shared codes for these encounters across all service lines. These findings are counter to the OIG report, and suggest that $<2$-midnight inpatients are different from observation patients at our institution, and that $<2$-midnight inpatients should not be arbitrarily reclassified as observation based solely upon LOS.

We also found that the majority of top ICD-9 codes within observation were the same regardless of LOS, suggesting that LOS does not reliably differentiate clinically different observation populations that merit different insurance coverage (Medicare Part A for $\geq 2$-midnight encounters, Medicare Part B for $<2$ midnight encounters). This lack of a clear cut point may drive an overall increase in LOS to achieve 2 midnights, as common diagnosis codes can be justified for both $<2$-midnight or $\geq 2$-midnight observation stays.

Finally, we found that external factors, such as the time of day and specific day (weekday vs weekend) of hospitalization, impact the likelihood of achieving a $\geq 2$-midnight stay. Patients hospitalized earlier in the day were less likely to span 2 midnights compared to later-day encounters, suggesting that use of a full working day as the day of presentation is harmful to a patient's chance of gaining inpatient status. Observation hospitalizations starting on a weekend day were more likely to achieve 2 midnights, which likely reflects different resource allocation and hospital efficiency on weekends, yet it is unlikely that weekend midnights, if associated with any delay in care, will be counted toward a patient's cumulative 2-midnight total. The CMS has further indicated that midnights accrued prior to transfer from 1 hospital to another will not count toward a cumulative 2-midnight stay. Although it would seem likely that patients requiring transfer to a tertiary care center would meet inpatient criteria, $9 \%$ of our observation encounters were transferred from other acute care hospitals, and many will have lost attributable midnights accrued prior to trans- fer to our hospital. Taken together, our findings strongly suggest that issues entirely unrelated to diagnosis or clinical status will impact whether hospitalized patients will be classified as inpatient or observation under the new rule.

This study has several limitations. Our data were limited to a single Midwestern tertiary care academic medical center, and may not be applicable to other healthcare settings. Second, $1.5 \%$ (466) of our inpatient stays and $56(1.2 \%)$ of our observation encounters lacked a referral source in our administrative database, although these deficiencies would not likely change the conclusions. Finally, the total number of observation encounters starting before 8:00 AM and staying $\geq 2$ midnights was small and therefore potentially subject to confounding. However, despite the fact that encounters beginning in the early morning hours may be different in ways unable to be measured in this study, encounter ICD-9 codes were similar regardless of time of day.

Despite these limitations, our study raises concerns about the impact of CMS's new time-driven observation rules on hospital practice patterns. We found distinctly different $<2$-midnight inpatient ICD-9 codes as compared to those for observation encounters. Reclassifying <2-midnight inpatients as observation may inappropriately shift the financial burden of acute hospitalizations to patients who were previously eligible to receive Medicare inpatient benefits. We also demonstrated a decrease in billable inpatient encounters under the new rules, which may be countered by changes in hospital and provider practice to drive more admissions over the 2-midnight threshold to avoid financial losses. These changes should be defensible under audit given the ambiguities of the rule we found in this study surrounding time of day of admission, weekend and transfer hospitalizations, and the fact that common observation ICD-9 codes are similar across LOS. Ironically, the unintended consequence of the new rule may be to drive up hospital LOS, reduce efficiency, and increase the overall cost of care.

Our findings suggest that CMS should define observation care per its original intent: as a means to determine if a patient can safely return home after a short period of additional care. ${ }^{6}$ Patients whose conditions necessitate an intensity and level of service beyond this narrow scope should be classified as full inpatients irrespective of LOS, whether that LOS is $<2$ midnights or $\geq 2$ midnights. Policies that fail to reflect the original intent of observation status are unlikely to achieve the intended outcome of defining 2 distinct patient populations that merit different services and reimbursement.

\section{Acknowledgements}

The authors thank Andrew LaRocque and Dan Dexter for their assistance with the data. Without their help, this article would not have been possible.

Disclosures: Dr. Graf reports receiving royalties from Smith \& Nephew for patents he has assigned to them involving orthopedic implants. This 
is unrelated to the topic or content of this manuscript. The authors report no other conflicts of interest.

Editor's Note: On February 24, 2014, after this manuscript was published, CMS issued a rules clarification allowing midnights spent at a referral hospital prior to transfer to be counted in the cumulative 2 midnight tally to determine inpatient status.

\section{References}

1. Feng Z, Wright B, Mor V. Sharp rise in Medicare enrollees being held hospitals for observation raises concerns about causes and consequences. Health Aff (Millwood). 2012;31(6):1251-1259.

2. MedPAC report to Congress: hospital inpatient and outpatient services. Available at: http://www.medpac.gov/chapters/Mar13_Ch03. pdf. Accessed September 29, 2013.

3. Sheehy AM, Graf B, Gangireddy S, et al. Hospitalized but not admitted: characteristics of patients with "Observation Status" at an academic medical center. JAMA Intern Med. 2013;173(21):1991-1998.

4. Centers for Medicare and Medicaid Services inpatient prospective payment system 1599-F. Fiscal year 2014 final rule. Available at: http://www.gpo.gov/fdsys/pkg/FR-2013-08-19/pdf/2013-18956.pdf. Accessed December 22, 2013.

5. McKesson Interqual. Available at: http://www.mckesson.com/en_us/ McKesson.com/Payers/Decision\%2BManagement/InterQual\%2BEvid ence-Based $\% 2$ BClinical $\% 2$ BContent/InterQual $\% 2 B E v i d e n c e-B a s e d \%$ 2BClinical\%2BContent.html. Accessed October 3, 2013.

6. Medicare benefit policy manual. Chapter 6. Hospital services covered under Part B. Available at: https://www.cms.gov/transmittals/downloads/R42BP.pdf. Accessed December 22, 2013.

7. Gesensway D. Thinking of admitting this patient? Think again: how observation care is complicating life for you (and your patients).
Today's Hospitalist. Available at: http://www.todayshospitalist.com/ index.php? $\mathrm{b}=$ articles_read\&cnt $=1434$. Published February 2012. Accessed September 29, 2013.

8. Department of Health and Human Services Office of Inspector General. Hospitals' use of observation stays and short inpatient stays for Medicare Beneficiaries, OEI-02-12-00040. Issued July 29, 2013. Available at: http://oig.hhs.gov/oei/reports/oei-02-12-00040.asp. Accessed September 29, 2013.

9. The Centers for Medicare and Medicaid Services frequently asked questions for 2 midnight inpatient admission guidance and patient status reviews for admissions on or after October 1, 2013. Available at: http://cms.gov/Research-Statistics-Data-and-Systems/Monitoring-Pro grams/Medicare-FFS-Compliance-Programs/Medical-Review/Downloads/ QuestionsandAnswersRelatingtoPatientStatusReviews_12232013_508Cle an.pdf. Accessed January 31, 2014.

10. Facts and figures: University of Wisconsin Hospital and Clinics. Available at: http://www.uwhealth.org/files/uwhealth/docs/pdf/uwhc_factsfigures.pdf. Accessed October 1, 2013.

11. Centers for Medicare and Medicaid Services. Reviewing hospital claims for patient status. Available at: http://cms.gov/Research-Statistics-Dataand-Systems/Monitoring-Programs/Medicare-FFS-Compliance-Programs/ Medical-Review/Downloads/ReviewingHospitalClaims_forAdmis sion_forPosting_01312014_508Clean.pdf. Accessed January 31, $201 \overline{4}$.

12. Sheehy AM, Graf B, Gangireddy S, Formisano R, Jacobs E. "Observation Status" for hospitalized patients: implications of a proposed Medicare rules change. JAMA Intern Med. 2013;173(21): 2004-2006

13. Kaiser Health News. HHS Inspector General scrutinizes Medicare observation care policy. Available at: http://www.kaiserhealthnews. org/stories/2013/july/30/ig-report-observation-care.aspx. Accessed October 3, 2013. 